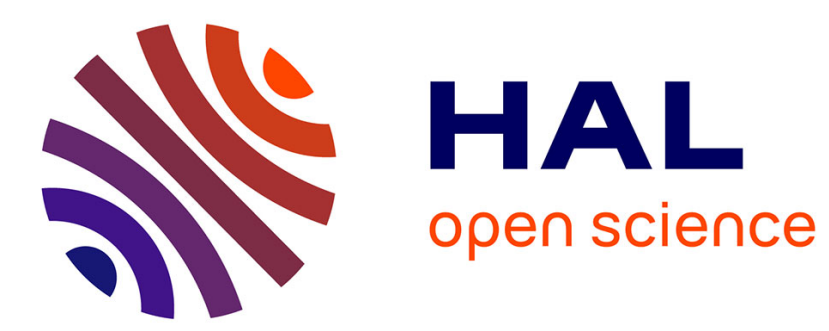

\title{
Approximation power of C1-smooth isogeometric splines on volumetric two-patch domains
}

\author{
Katharina Birner, Bert Jüttler, Angelos Mantzaflaris
}

\section{To cite this version:}

Katharina Birner, Bert Jüttler, Angelos Mantzaflaris. Approximation power of C1-smooth isogeometric splines on volumetric two-patch domains. IGAA 2018, Apr 2018, Delft, Netherlands. hal-02275654

\section{HAL Id: hal-02275654 \\ https://hal.inria.fr/hal-02275654}

Submitted on 1 Sep 2019

HAL is a multi-disciplinary open access archive for the deposit and dissemination of scientific research documents, whether they are published or not. The documents may come from teaching and research institutions in France or abroad, or from public or private research centers.
L'archive ouverte pluridisciplinaire HAL, est destinée au dépôt et à la diffusion de documents scientifiques de niveau recherche, publiés ou non, émanant des établissements d'enseignement et de recherche français ou étrangers, des laboratoires publics ou privés. 


\title{
Approximation power of $C^{1}$-smooth isogeometric splines on volumetric two-patch domains
}

\author{
Katharina Birner ${ }^{\dagger}$, Bert Jüttler ${ }^{\dagger}$, and Angelos Mantzaflaris ${ }^{\dagger, \dagger}$
}

\begin{abstract}
Bases and dimensions of trivariate spline functions possessing first order geometric continuity on two-patch domains were studied in [4]. It was shown that the properties of the spline space depend strongly on the type of the gluing data that is used to specify the relation between the partial derivatives along the interface between the patches. Locally supported bases were shown to exist for trilinear geometric gluing data (that corresponds to piecewise trilinear domain parameterizations) and sufficiently high degree. The present paper is devoted to the approximation properties of these spline functions.

We recall the construction of the basis functions and show how to compute them efficiently. In contrast to the results in [4], which relied on exact arithmetic operations in the field of rational numbers, we evaluate the coefficients by computations with standard floating point numbers. We then perform numerical experiments with $L^{2}$-projection in order to explore the approximation power of the resulting spline functions. Despite the existence of locally supported bases, we observe a reduction of the approximation order for low degrees, and we provide a theoretical explanation for this locking.
\end{abstract}

\section{Introduction}

The framework of Isogeometric Analysis [7] facilitates numerical simulation with high-order partial differential equations, since it supports $C^{r}$-smooth discretizations with $r>0$. For $r=1$, these are especially useful when considering PDEs of order four, such as the Cahn-Hilliard equation [8], shells [2], and the biharmonic equation [1].

\footnotetext{
${ }^{\dagger}$ Institute of Applied Geometry, Johannes Kepler University, Altenberger Straße 69, 4040 Linz, Austria. ${ }^{\ddagger}$ Université Côte d'Azur, Inria Sophia Antipolis - Méditerranée, 2004 route des Lucioles - B.P. 93, France. E-mails: \{katharina.birner|bert.juettler\}@jku.at, angelos.mantzaflaris@inria.fr
} 
While the construction of $C^{1}$-smooth spline functions on single patches is straightforward, the extension to multi-patch domains, which are needed to describe more complex domains, requires the notion of geometric continuity [16]. More precisely, $C^{r}$-smoothness $(r \geq 0)$ of an isogeometric spline function is implied by $G^{r}$ smoothness (geometric continuity of order $r$ ) of the associated graph surface [9]. This result has widely been used to construct $C^{1}$-smooth spline spaces on planar domains.

Smooth approximations over unstructured quadrilateral meshes were considered in [3]. The construction of geometrically continuous splines on arbitrary topologies was studied in [14]. Bases and dimensions of the space of $C^{1}$-smooth isogeometric functions for bilinear parameterized domains were explored in [10]. Some of these results have been extended to $C^{2}$-smooth splines [12].

The numerical examples presented in these publications indicate optimal approximation power for combinations of sufficiently high degrees with certain classes of gluing data. In particular, the generalization of parameterizations with bilinear gluing data to the more general class of analysis-suitable parameterizations, which appears to preserve the optimal approximation properties, was presented in $[6,11]$.

The extension to trivariate domains was studied in $[4,5,15]$. The domains considered in [15] are obtained via sweeping, which restricts the available topologies. In [4], we studied the space of globally $C^{1}$-smooth splines on a two-patch domain, which is topologically equivalent to two cuboids. We considered different types of gluing data and presented the associated dimension formulas. Moreover, we showed how to construct a basis and identified those types of gluing that yield locally supported basis functions indicating good approximation properties.

The space of $C^{1}$-smooth isogeometric functions for trilinearly parameterized two-patch domains was further studied in [5]. Explicit representations of the locally supported basis functions were presented and the numerically obtained dimension formula from [4] was theoretically verified.

The present paper extends these existing results. In Section 2, we recall the notion of the glued spline space $\mathfrak{G}_{D}$, which characterizes the space of $C^{1}$-smooth isogeometric functions $\mathscr{V}_{\boldsymbol{F}}$. Based on these preparations, Section 3 considers the coefficient patterns of trilinear geometric gluing data, which was found to be promising for good approximation power in [4], in further detail. These patterns allow us to efficiently compute a basis of the space $\mathscr{V}_{\boldsymbol{F}}$ for this type of gluing data. Finally, in Section 4 we numerically analyze the approximation power of the basis via $L^{2}$ fitting. We conclude the paper in Section 5.

\section{Preliminaries}

We consider two subdomains $\Omega^{(1)}$ and $\Omega^{(2)}$, both topologically equivalent to a cube, which form the two-patch geometry $\Omega=\Omega^{(1)} \cup \Omega^{(2)}$. Let $\mathscr{S}_{k}^{p}$ denote the space of spline functions on $[0,1]$ of degree $p$ with $k$ uniformly distributed inner knots of multiplicity $p-1$. We use it to define the tensor-product space 


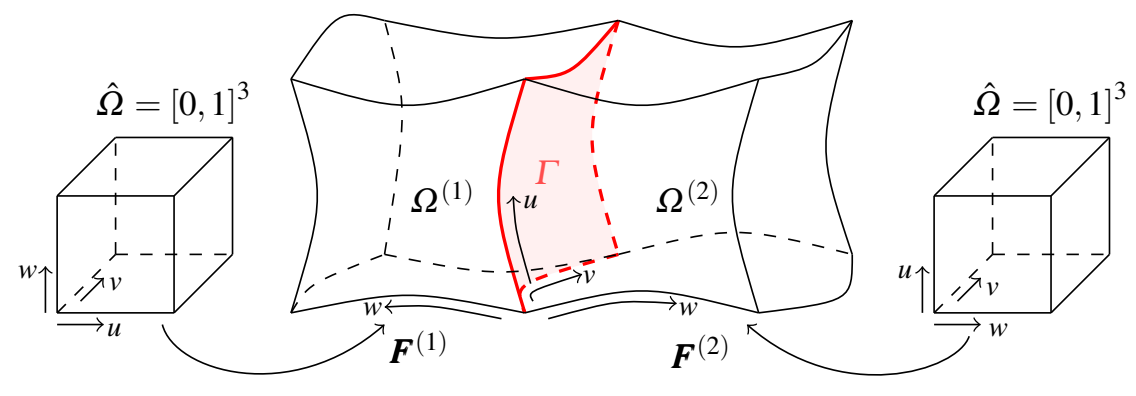

Fig. 1: Parameterizations of two volumetric subdomains $\Omega^{(1)}$ and $\Omega^{(2)}$ joined at the interface $\Gamma$.

$$
\mathscr{P}=\mathscr{S}_{k}^{p} \otimes \mathscr{S}_{k}^{p} \otimes \mathscr{S}_{k}^{p}
$$

which consists of $C^{1}$-smooth trivariate spline functions.

The two subdomains are described by parametric representations $\boldsymbol{F}^{(i)}: \hat{\Omega}=$ $[0,1]^{3} \rightarrow \Omega^{(i)}, i=1,2$ with coordinate functions from $\mathscr{P}$, see Figure 1 . These define the two-patch geometry mapping

$$
\boldsymbol{F}=\left(\boldsymbol{F}^{(1)}, \boldsymbol{F}^{(2)}\right) \in \mathscr{P}^{3} \times \mathscr{P}^{3} .
$$

We assume that the two patches meet with $C^{0}$-smoothness

$$
\boldsymbol{F}^{(1)}(u, v, 0)=\boldsymbol{F}^{(2)}(u, v, 0), \quad u, v \in[0,1]
$$

at the common interface $\Gamma=[0,1]^{2} \times\{0\}$. In this paper, we explore the space

$$
\mathscr{V}_{\boldsymbol{F}}=\left[(\mathscr{P} \times \mathscr{P}) \circ \boldsymbol{F}^{-1}\right] \cap C^{1}\left(\Omega^{(1)} \cup \Omega^{(2)}\right)
$$

of $C^{1}$-smooth isogeometric functions on $\Omega$.

The elements of the pairs $\boldsymbol{f}=\left(f^{(1)}, f^{(2)}\right) \in \mathscr{P} \times \mathscr{P}$ possess representations

$$
f^{(i)}=\sum_{k=0}^{n} b_{k}^{(i)} N_{k}(u, v, w), \quad i=1,2,
$$

with real coefficients $b_{k}^{(i)}$, where the symbols $N_{k}$ denote the tensor-product B-splines that span the space $\mathscr{P}$. The glued spline space $\mathfrak{G}_{D}$ is a subspace of $\mathscr{P} \times \mathscr{P}$, which was introduced in [4]. For given gluing data $D=\left(\beta, \gamma, \alpha^{(1)}, \alpha^{(2)}\right)$, which is a quadruple of four bivariate polynomials, it consists of all functions whose coefficients satisfy 


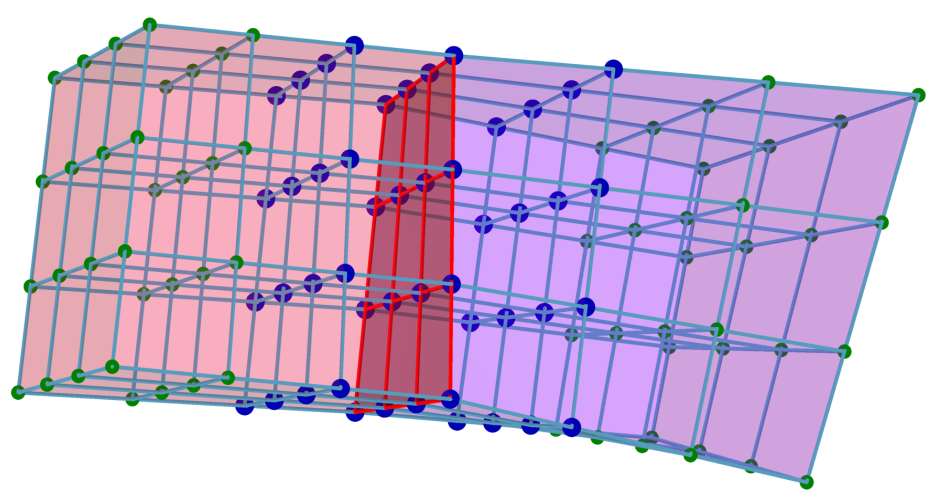

Fig. 2: Coefficients used by the interface functions (blue bullets) and coefficients contributing to inner functions (green bullets). The figure shows the control cages of both patches.

$$
\begin{aligned}
0= & \sum_{k=0}^{n} b_{k}^{(1)} N_{k}(u, v, 0)-b_{k}^{(2)} N_{k}(u, v, 0) \quad \text { and } \\
0= & \sum_{k=0}^{n} b_{k}^{(1)}\left(\beta(u, v)\left(\partial_{u} N_{k}\right)(u, v, 0)-\gamma(u, v)\left(\partial_{v} N_{k}\right)(u, v, 0)\right. \\
& \left.\quad+\alpha^{(1)}(u, v)\left(\partial_{w} N_{k}\right)(u, v, 0)\right)-b_{k}^{(2)} \alpha^{(2)}(u, v)\left(\partial_{w} N_{k}\right)(u, v, 0) .
\end{aligned}
$$

Bases and dimensions of the glued spline space $\mathfrak{G}_{D}$ for different types of gluing data were studied in [4]. Furthermore it was observed that

$$
\mathscr{V}_{\boldsymbol{F}}=\mathfrak{G}_{D} \circ \boldsymbol{F}^{-1}
$$

for regular geometry mappings $\boldsymbol{F} \in \mathfrak{G}_{D}$. This means that any $C^{1}$-smooth isogeometric function is the push-forward of a glued spline function. This result is closely related to [9], which establishes the fact that matched $G^{k}$-constructions always yield $C^{k}$-continuous isogeometric elements in a more general setting.

Following the approach in [4], we construct a basis of the space $\mathscr{V}_{F}$ - and consequently of the space $\mathfrak{G}_{D}$ - by splitting the space into a direct sum of two subspaces, i.e.

$$
\mathscr{V}_{\boldsymbol{F}}=\mathscr{V}_{\boldsymbol{F}}^{\Gamma} \oplus \mathscr{V}_{\boldsymbol{F}}^{S}
$$

The first subspace $\mathscr{V}_{\boldsymbol{F}}^{\Gamma}$ denotes the space of interface functions. It contains the functions with non-zero coefficients on the shared face $\Gamma$ and the two neighboring layers, see blue points in Figure 2. These functions are affected by the specific choice of gluing data concerned. The second subspace $\mathscr{V}_{F}^{S}$, referred to as the space of standard functions, contains functions with zero coefficients on these three layers, see the green bullets in Figure 2. It is spanned by the "usual" isogeometric basis functions and therefore does not depend on the choice of gluing data. 


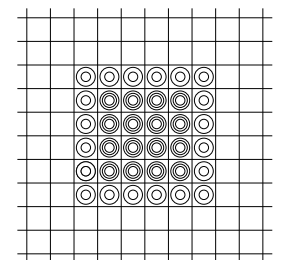

(a) Type 3

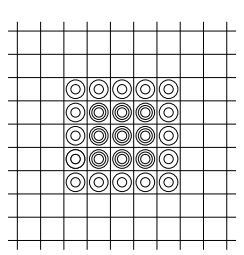

(e) Type 4.3

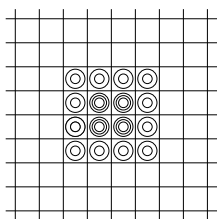

(b) Type 4.1

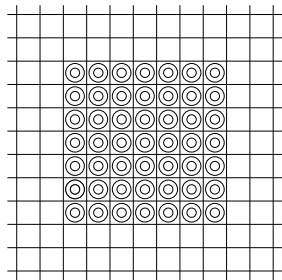

(f) Type 4.4

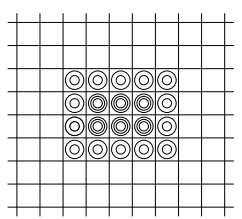

(c) Type 4.2.1

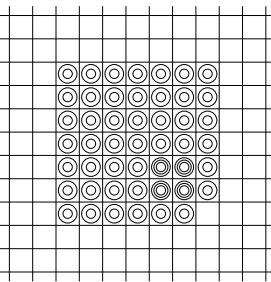

(g) Type 4.5

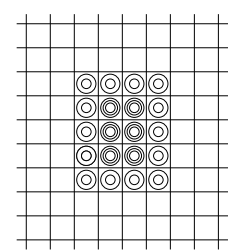

(d) Type 4.2.2

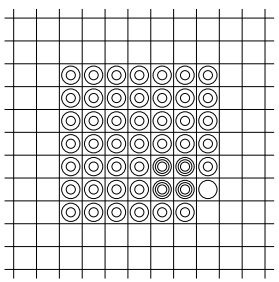

(h) Type 4.6

Fig. 3: Coefficient patterns of basis functions for trilinear geometric gluing data.

In order to keep the presentation concise, we restrict ourselves to spaces satisfying first order homogeneous boundary conditions along $\partial \Omega$, which is indicated by the subscript 0 , and we obtain the decomposition

$$
\mathscr{V}_{\boldsymbol{F}, 0}=\mathscr{V}_{\boldsymbol{F}, 0}^{\Gamma} \oplus \mathscr{V}_{\boldsymbol{F}, 0}^{S}
$$

\section{Basis construction}

As noted in [4], trilinear geometric gluing data is particularly promising for applications. We describe the construction of a basis for $\mathscr{V}_{\boldsymbol{F}, 0}^{\Gamma}$ in this case.

This type of gluing data is derived from a trilinear geometry mapping $\boldsymbol{F}=$ $\left(\boldsymbol{F}^{(1)}, \boldsymbol{F}^{(2)}\right)$, which is assumed to be regular. More precisely, it consists of the four polynomials

$$
\begin{array}{r}
\beta(u, v)=\operatorname{det}\left(\partial_{2} \boldsymbol{F}^{(1)}(u, v, 0), \partial_{3} \boldsymbol{F}^{(1)}(u, v, 0), \partial_{3} \boldsymbol{F}^{(2)}(u, v, 0)\right), \\
\gamma(u, v)=\operatorname{det}\left(\partial_{1} \boldsymbol{F}^{(1)}(u, v, 0), \partial_{3} \boldsymbol{F}^{(1)}(u, v, 0), \partial_{3} \boldsymbol{F}^{(2)}(u, v, 0)\right), \\
\alpha^{(1)}(u, v)=\operatorname{det} \nabla \boldsymbol{F}^{(2)}(u, v, 0), \\
\alpha^{(2)}(u, v)=\operatorname{det} \nabla \boldsymbol{F}^{(1)}(u, v, 0),
\end{array}
$$

which have bi-degrees $[(3,2),(2,3),(2,2),(2,2)]$. For $p \geq 3$, the dimension of the space $\mathscr{V}_{\boldsymbol{F}, 0}^{\Gamma}$ is equal to 


$$
\operatorname{dim} \mathscr{V}_{\boldsymbol{F}, 0}^{\Gamma}=10+k(2-11 k)-p\left(2+2 k-4 k^{2}\right) .
$$

Locally supported basis functions for trilinear geometric gluing data were presented in [4]. For the sake of completeness, we recall the obtained coefficient patterns in Figure 3 . The basis for degree $p=3$ is obtained by performing index shifts in $(2 \mathbb{Z})^{2}$ for Type 3 , while the basis for $p=4$ consists of seven different types:

- $2 k-1$ functions of Type 4.1 , with shifts in $2 \cdot 3 \mathbb{Z}$,

- $k-1$ functions of Type 4.2.1, with shifts in $3 \mathbb{Z}$,

- $k(k-1)$ functions of Type 4.2.2, with shifts in $(3 \mathbb{Z})^{2}$, and

- $(k-1)^{2}$ functions of Types 4.3-4.6, with shifts in $(3 \mathbb{Z})^{2}$.

The coefficient patterns of the basis functions described above were found by studying the kernel of the matrix formed by collocating the equation (1) at suitable Greville abscissa. The first order homogeneous boundary conditions are incorporated by imposing additional constraints. The corank of the resulting matrix reveals the dimension of the spline space $\mathscr{V}_{\boldsymbol{F}, 0}$, and consequently also of the subspace $\mathscr{V}_{\boldsymbol{F}, 0}^{\Gamma}$. Repeated patterns were observed in suitably constructed kernel vectors of that matrix, which allowed us to derive local subproblems that yield a single basis function.

To point out the importance of these local patterns, note that the computation of the sparsest kernel vectors (that is, the functions with the smallest possible support) is NP-hard. Therefore, even for small numbers of inner knots $k$, the computations can be rather inefficient. Another issue is that computing the rank and the corank of a matrix with floating point numbers can only be done up to certain precision. This can create some ambiguity or uncertainty on the actual dimension of the spline space.

In [4], the first problem was addressed by manually designing suitable orderings of the coefficients that lead to sparse coefficient patterns when performing RREF ${ }^{1}$ computations. We dealt with the second issue by using rational arithmetic. In particular, the matrix obtained from (1) has rational elements, since they are evaluations of piecewise polynomial functions with rational coefficients at rational Greville points. However, as the dimension of the problem increases, working with rational arithmetic becomes prohibitive.

In the present work, we exploit the fact that the local subproblems, which are defined by the shifted coefficient patterns, are known to have a kernel dimension equal to one. Consequently, it is no longer necessary to use rational arithmetic. Instead, since we know that we are looking for a single kernel vector, we use floating point computations and perform singular value decompositions. We then keep the vector associated to the singular value closest to zero.

The savings in time and memory needed for the basis computation when using floating point operations instead of exact arithmetic are presented in Table 1 and Table 2. The entries of the tables refer to the construction of the basis of the space $\mathscr{V}_{\boldsymbol{F} .0}^{\Gamma}$ for spline degree $p=3$ and different numbers of inner knots $k$. We compare the approach used in [4], where we had to set up the complete matrix obtained

\footnotetext{
${ }^{1}$ Reduced Row Echelon Form
} 


\begin{tabular}{c|cccc} 
& $k=3$ & $k=7$ & $k=15$ & $k=31$ \\
\hline Floating point & $0.01 \mathrm{~s}$ & $0.07 \mathrm{~s}$ & $1.03 \mathrm{~s}$ & $25.82 \mathrm{~s}$ \\
Rational & $5.73 \mathrm{~s}$ & $1037.56 \mathrm{~s}$ & $>24 \mathrm{~h}$ & $>72 \mathrm{~h}$
\end{tabular}

Table 1: Time needed for constructing the basis using floating point and rational computations.

\begin{tabular}{c|cccc} 
& $k=3$ & $k=7$ & $k=15$ & $k=31$ \\
\hline Floating point & 9.9 MBytes & 11.4 MBytes & 17.6 MBytes & 42.4MBytes \\
Rational & 20.8MBytes & 426.8MBytes & >17.7GBytes & >32GBytes ${ }^{2}$
\end{tabular}

Table 2: Memory needed for constructing the bases using floating point and rational computations.

from (1) using rational arithmetic, with the more efficient construction using local subproblems and SVD based on floating point operations.

The entries in Table 1 show the time spent on the computations, whereas the values in Table 2 depict the maximal resident set size (RSS), which is the amount of memory occupied by the computation that is held in main memory (RAM). The expected massive advantage of the localized computation using floating point operations is clearly visible.

\section{Approximation properties}

We explore the approximation power of the basis obtained for trilinear geometric gluing data in case of spline degree $p=3,4$. To determine the rates of convergence we use $L^{2}$-fitting of suitable target functions that are defined on the two-patch geometry shown in Figure 4. Besides the usual norms, which are defined on the entire domain, we analyze the residuals via the following norms on the interface:

\begin{tabular}{c||c|c|c} 
Type & $H^{1}(\Gamma)$ & $L^{2}(\Gamma)$ & $L^{\infty}(\Gamma)$ \\
\hline Norm & $\left\|\left.\nabla\left(f_{\Gamma} \circ \boldsymbol{F}^{-1}\right)\right|_{\Gamma}\right\|_{2}$ & $\sqrt{\int_{\Gamma}\left|f_{\Gamma} \circ \boldsymbol{F}^{-1}\right|^{2} d s}$ & $\max _{\Gamma}\left|f_{\Gamma} \circ \boldsymbol{F}^{-1}\right|$
\end{tabular}

with $f_{\Gamma}=\left.f^{(1)}\right|_{\Gamma}=\left.f^{(2)}\right|_{\Gamma}$.

For bivariate $C^{1}$-smooth spline spaces, optimal convergence rates were obtained for degree $p=3$ and higher, see [13]. However, this does not extend to the trivariate case of trilinearly parameterized two-patch domains, as shown in Figure 5. In the left picture, the global error is shown, where a small reduction in the approximation power can be recognized. This loss in the convergence rate is solely introduced by the error on the interface, which is depicted in the right plot.

The reduction can be explained by taking a closer look on the involved spline functions. Consider two spline functions $f, \hat{f} \in \mathfrak{G}_{D}$, with $\left.f\right|_{\Gamma}=\left.\hat{f}\right|_{\Gamma}$. Since both

\footnotetext{
${ }^{2}$ Aborted because of too high memory requirements.
} 


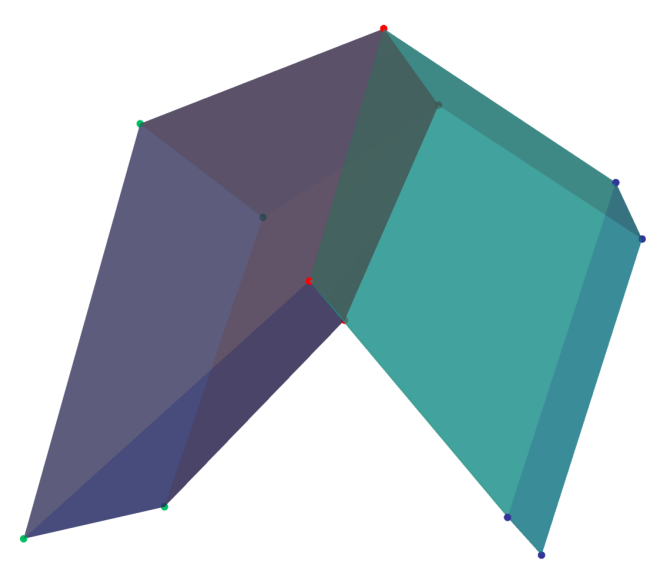

Fig. 4: Two-patch geometry used for $L^{2}$-fitting.

functions are elements of the glued spline space $\mathfrak{G}_{D}$, they satisfy the following equation

$$
\beta \partial_{u} g^{(1)}-\gamma \partial_{v} g^{(1)}+\alpha^{(1)} \partial_{w} g^{(1)}-\alpha^{(2)} \partial_{w} g^{(2)}=0,
$$

with $g \in \mathfrak{G}_{D}, g^{(1)}=\left.g\right|_{\Omega^{(1)}}, g^{(2)}=\left.g\right|_{\Omega^{(2)}}$, see (1).

Therefore, their difference, which is again a function in the glued spline space, satisfies

$$
\begin{aligned}
& \beta \underbrace{\left.\partial_{u}\left(f^{(1)}-\hat{f}^{(1)}\right)\right|_{\Gamma}}_{=0}-\gamma \underbrace{\left.\partial_{v}\left(f^{(1)}-\hat{f}^{(1)}\right)\right|_{\Gamma}}_{=0}+ \\
& \left.\alpha^{(1)} \partial_{w}\left(f^{(1)}-\hat{f}^{(1)}\right)\right|_{\Gamma}-\left.\alpha^{(2)} \partial_{w}\left(f^{(2)}-\hat{f}^{(2)}\right)\right|_{\Gamma}=0,
\end{aligned}
$$

hence

$$
\left.\alpha^{(1)} \partial_{w}\left(f^{(1)}-\hat{f}^{(1)}\right)\right|_{\Gamma}=\left.\alpha^{(2)} \partial_{w}\left(f^{(2)}-\hat{f}^{(2)}\right)\right|_{\Gamma}
$$

It has been observed that $\operatorname{gcd}\left(\alpha^{(1)}, \alpha^{(2)}\right)=1$, see [5]. This implies that $\alpha^{(1)}$, which is a bivariate polynomial of degree $(2,2)$, is a factor of each of the polynomial segments of

$$
d^{(2)}=\left.\partial_{w}\left(f^{(2)}-\hat{f}^{(2)}\right)\right|_{\Gamma} .
$$

Similarly, $\alpha^{(2)}$ is a factor of each of the polynomial segments of

$$
d^{(1)}=\left.\partial_{w}\left(f^{(1)}-\hat{f}^{(1)}\right)\right|_{\Gamma} .
$$

We obtain two $C^{1}$-smooth piecewise polynomial functions $d^{(1)} / \alpha^{(2)}$ and $d^{(2)} / \alpha^{(1)}$. Since the degree of these functions does not exceed $(3,3)-(2,2)=(1,1)$, we conclude that the spline functions

$$
d^{(i)}=\left.\partial_{w}\left(f^{(i)}-\hat{f}^{(i)}\right)\right|_{\Gamma}, \quad i=1,2
$$



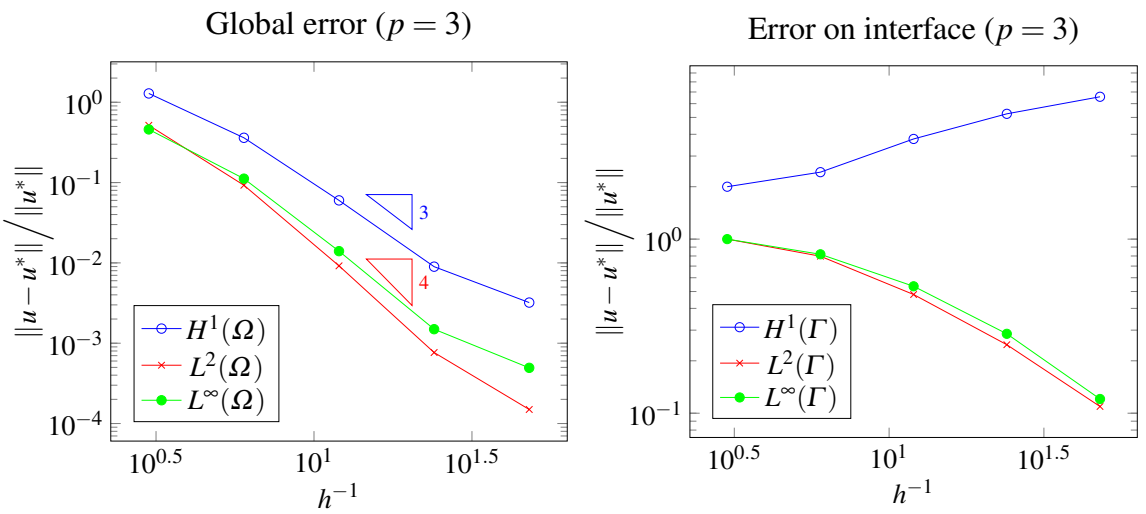

\begin{tabular}{lllllll}
\hline $\mathrm{h}$ & $H^{1}(\Omega)$ & $L^{2}(\Omega)$ & $L^{\infty}(\Omega)$ & $H^{1}(\Gamma)$ & $L^{2}(\Gamma)$ & $L^{\infty}(\Gamma)$ \\
\hline 0.33 & 1.29 & 0.52 & 0.46 & 2 & 1 & 1 \\
0.17 & 0.36 & $9.27 \cdot 10^{-2}$ & 0.11 & 2.42 & 0.8 & 0.82 \\
$8.33 \cdot 10^{-2}$ & $6.00 \cdot 10^{-2}$ & $9.19 \cdot 10^{-3}$ & $1.40 \cdot 10^{-2}$ & 3.76 & 0.48 & 0.54 \\
$4.17 \cdot 10^{-2}$ & $8.97 \cdot 10^{-3}$ & $7.66 \cdot 10^{-4}$ & $1.50 \cdot 10^{-3}$ & 5.24 & 0.25 & 0.29 \\
$2.08 \cdot 10^{-2}$ & $3.21 \cdot 10^{-3}$ & $1.50 \cdot 10^{-4}$ & $4.94 \cdot 10^{-4}$ & 6.57 & 0.11 & 0.12 \\
\hline
\end{tabular}

Fig. 5: $L^{2}$-approximation errors for trilinear geometric gluing data of degree $p=3$.

are indeed single polynomials of degree $(3,3)$ and therefore $C^{\infty}$-smooth.

Consequently, the cross-boundary derivatives of any two functions $f$ and $\hat{f}$, which take the same values on the interface $\Gamma$, differ only by a bi-cubic polynomial with only four degrees of freedom, and this does not change as $h$ is decreased. This observation, which is in agreement with the results (that were obtained by a slightly different approach) in [5], explains the loss of approximation power, as follows.

We consider two smooth functions $\varphi, \hat{\varphi} \in C^{\infty}(\Omega)$. There exist two sequences $\left(f_{h}\right)_{h}$ and $\left(\hat{f}_{h}\right)_{h}$ of trivariate tensor-product spline functions with uniform knots, whose elements are taken from the glued spline spaces obtained for element size

$$
h=\frac{1}{k+1} \rightarrow 0,
$$

such that $\left(f_{h} \circ \boldsymbol{F}^{-1}\right)_{h}$ and $\left(\hat{f}_{h} \circ \boldsymbol{F}^{-1}\right)_{h}$ converge to $\varphi$ and $\hat{\varphi}$, respectively. If these sequences converged with the full approximation power, the derivatives would converge as well, hence

$$
d_{h}^{(i)}=\left.\left.\partial_{w}\left(f_{h}^{(i)}-\hat{f}_{h}^{(i)}\right)\right|_{\Gamma} \rightarrow\left(\partial_{w}\left((\varphi-\hat{\varphi}) \circ \boldsymbol{F}^{(i)}\right)\right)\right|_{\Gamma}, \quad i=1,2,
$$

as $h \rightarrow 0$. However, this is impossible for almost all pairs of given functions $\varphi, \hat{\varphi}$, since the functions $d_{h}^{(i)}$ are single bicubic polynomials for any $h$. 

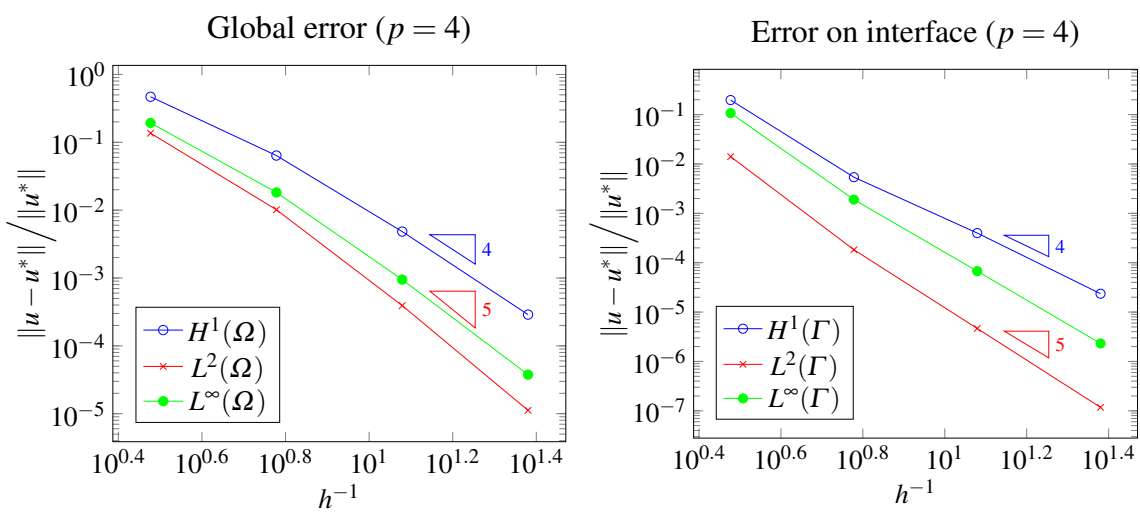

\begin{tabular}{lllllll}
\hline $\mathrm{h}$ & $H^{1}(\Omega)$ & $L^{2}(\Omega)$ & $L^{\infty}(\Omega)$ & $H^{1}(\Gamma)$ & $L^{2}(\Gamma)$ & $L^{\infty}(\Gamma)$ \\
\hline 0.33 & 0.47 & 0.14 & 0.19 & 0.2 & $1.41 \cdot 10^{-2}$ & 0.11 \\
0.17 & $6.38 \cdot 10^{-2}$ & $1.02 \cdot 10^{-2}$ & $1.83 \cdot 10^{-2}$ & $5.40 \cdot 10^{-3}$ & $1.83 \cdot 10^{-4}$ & $1.90 \cdot 10^{-3}$ \\
$8.33 \cdot 10^{-2}$ & $4.83 \cdot 10^{-3}$ & $3.92 \cdot 10^{-4}$ & $9.49 \cdot 10^{-4}$ & $3.99 \cdot 10^{-4}$ & $4.66 \cdot 10^{-6}$ & $6.75 \cdot 10^{-5}$ \\
$4.17 \cdot 10^{-2}$ & $2.89 \cdot 10^{-4}$ & $1.12 \cdot 10^{-5}$ & $3.76 \cdot 10^{-5}$ & $2.36 \cdot 10^{-5}$ & $1.17 \cdot 10^{-7}$ & $2.31 \cdot 10^{-6}$ \\
\hline
\end{tabular}

Fig. 6: $L^{2}$-approximation errors for trilinear geometric gluing data of degree $p=4$.

If we consider spline degrees $p>3$, this argument no longer applies and we observe full approximation power, as shown in Figure 6 for $p=4$.

\section{Conclusion}

Based on earlier results about locally supported bases on trivariate two-patch domains, which were shown to exist for trilinear geometric gluing data (that corresponds to piecewise trilinear domain parameterizations) and sufficiently high degree, we investigated the approximation properties of these functions. In addition we also showed how to efficiently compute the basis functions using standard arithmetic (i.e., floating point numbers). We observed that the existence of locally supported interface basis functions for spline degree $p=3$ does not suffice to provide optimal approximation power, even though these functions take non-zero values along the interface. In addition to the experimental results we also derived a theoretical justification for this surprising fact. We also confirmed that these effects are no longer present for higher polynomial degrees. Future work will be devoted to multi-patch domains with more than two patches and to applications in numerical simulation. 


\section{Acknowledgment}

Supported by the Austrian Science Fund (FWF) through NFN S117 "Geometry + Simulation". The authors thank the reviewer for the constructive comments.

\section{References}

1. A. Bartezzaghi, L. Dedè, and A. Quarteroni. Isogeometric analysis of high order partial differential equations on surfaces. Comput. Methods Appl. Mech. Engrg., 295:446 - 469, 2015.

2. L. Beirao da Veiga, A. Buffa, C. Lovadina, M. Martinelli, and G. Sangalli. An isogeometric method for the Reissner-Mindlin plate bending problem. Comput. Methods Appl. Mech. Engrg., 209:45-53, 2012.

3. M. Bercovier and T. Matskewich. Smooth Bézier Surfaces over Unstructured Quadrilateral Meshes. Lecture Notes of the Unione Matematica Italiana, Springer, 2017.

4. K. Birner, B. Jüttler, and A. Mantzaflaris. Bases and dimensions of $C^{1}$-smooth isogeometric splines on volumetric two-patch domains. Graph. Mod., 99:46-56, 2018.

5. K. Birner and M. Kapl. The space of $C^{1}$-smooth isogeometric spline functions on trilinearly parameterized volumetric two-patch domains. Comput. Aided Geom. Des., 70:16-30, 2019.

6. A. Collin, G. Sangalli, and T. Takacs. Analysis-suitable $\mathrm{G}^{1}$ multi-patch parametrizations for $\mathrm{C}^{1}$ isogeometric spaces. Comput. Aided Geom. Des., 47:93 - 113, 2016.

7. J. A. Cottrell, T. Hughes, and Y. Bazilevs. Isogeometric Analysis: Toward Integration of CAD and FEA. John Wiley \& Sons, Chichester, England, 2009.

8. L. Dedè, M. J. Borden, and T. J. Hughes. Isogeometric analysis for topology optimization with a phase field model. Arch. Comput. Methods Engrg., 19:427-465, 2012.

9. D. Groisser and J. Peters. Matched $\mathrm{G}^{k}$-constructions always yield $\mathrm{C}^{k}$-continuous isogeometric elements. Comput. Aided Geom. Des., 34:67 - 72, 2015.

10. M. Kapl, F. Buchegger, M. Bercovier, and B. Jüttler. Isogeometric analysis with geometrically continuous functions on planar multi-patch geometries. Comput. Methods Appl. Mech. Engrg., 316:209-234, 2017.

11. M. Kapl, G. Sangalli, and T. Takacs. Construction of analysis-suitable $\mathrm{G}^{1}$ planar multi-patch parameterizations. Comput. Aided Des., 97:41-55, 2018.

12. M. Kapl and V. Vitrih. Space of $C^{2}$-smooth geometrically continuous isogeometric functions on planar multi-patch geometries: Dimension and numerical experiments. Comput. Math. Appl., 73(10):2319--2338, 2017.

13. M. Kapl, V. Vitrih, B. Jüttler, and K. Birner. Isogeometric analysis with geometrically continuous functions on two-patch geometries. Comput. Math. Appl., 70(7):1518 - 1538, 2015.

14. B. Mourrain, R. Vidunas, and N. Villamizar. Dimension and bases for geometrically continuous splines on surfaces of arbitrary topology. Comput. Aided Geom. Des., 45:108 - 133, 2016.

15. T. Nguyen, K. Karčiauskas, and J. Peters. $C^{1}$ finite elements on non-tensor-product $2 \mathrm{~d}$ and $3 \mathrm{~d}$ manifolds. Appl. Math. and Comp., 272:148 - 158, 2016.

16. J. Peters. Geometric continuity. In Handbook of computer aided geometric design, pages 193-227. North-Holland, Amsterdam, 2002. 